\title{
Inner and outer star forming regions over the discs of spiral galaxies I. Sample characterization
}

\author{
Marina Rodríguez-Baras ${ }^{1} \dagger$, A.I. Díaz ${ }^{1}$ and F.F. Rosales-Ortega ${ }^{2}$ \\ ${ }^{1}$ Departamento de Física Teórica, Universidad Autónoma de Madrid, 28049 Madrid, Spain. \\ ${ }^{2}$ Instituto Nacional de Astrofísica, Óptica y Electrónica, Luis E. Erro 1, 72840 Tonantzintla, \\ Puebla, Mexico
}

\begin{abstract}
This project is aimed at understanding the dependence of star formation on the environment by analysing young stellar populations in two very different positions in disk galaxies: circumnuclear and outer disk giant regions. Integral field spectroscopy (IFS) provide an ideal means to achieve these goals providing simultaneous spatial and spectral resolution. Here we present the characterization of the work sample, composed by 671 outer regions and 725 inner regions from 263 isolated spirals galaxies observed by the CALIFA survey. The wide number of regions in both samples allows us to obtain statistically relevant results about the influence of metallicity, density and environment on star formation, and how it disseminates over the galaxy, to obtain evolutionary stories for the star-forming regions and to compare our results with models of massive star formation and galactic chemical evolution.
\end{abstract}

Keywords. galaxies: general, galaxies: spiral, HII regions, stars: formation

\section{First results}

From 350 galaxies from the CALIFA survey (Sánchez et al. 2012) observed until october 2014 , we select the isolated spirals, obtaining a sample of 263 galaxies. We consider outer regions as those located at more than $2 R_{\text {eff }}$ to the galaxy center, and inner regions are those that fulfil the criterium established by Álvarez-Álvarez et al. (2015). Valid regions were selected applying quality criteria to ensure physical HII regions and avoid selection uncertainties. Some graphics showing first analysis are shown in Fig.1, and the whole characterization of the sample and a wider analysis will be published in the near future.
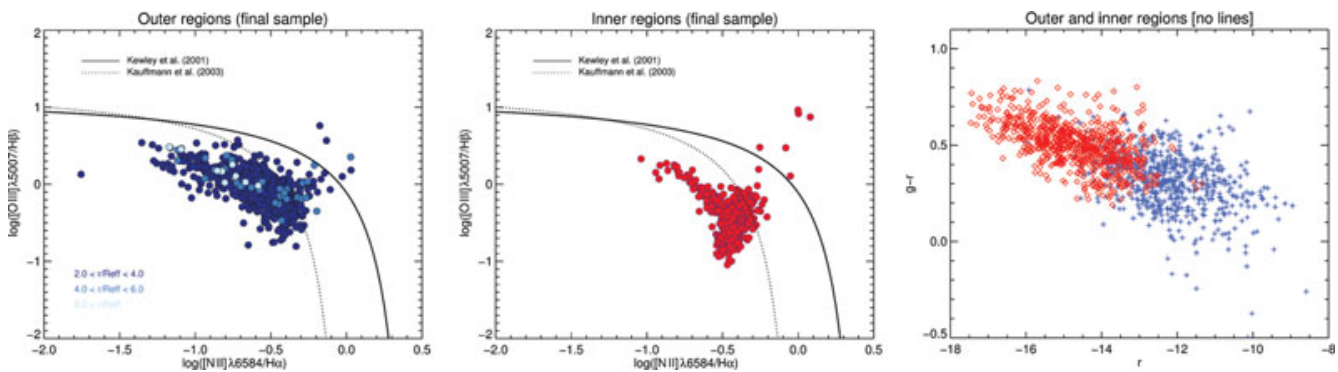

Figure 1. Left: Diagnostic diagram of the outer regions. Different colors represent different bins of distance to the center of the galaxy. Center: Diagnostic diagram of the inner regions. Right: Distribution of the inner (red, diamonds) and outer (blue, crosses) region samples in the g-r vs. $M_{\mathrm{r}}$ color-magnitude diagram. 